\title{
Harnessing Ultrasound-Stimulated Phase Change Contrast Agents to Improve Antibiotic Efficacy Against Methicillin-Resistant Staphylococcus aureus Biofilms
}

\author{
Phillip G. Durham 1,2, Ashelyn E. Sidders3, Paul A. Dayton 1,2, Brian P. Conlon 3,4 , Virginie \\ Papadopoulou $1^{*}$, Sarah E. Rowe ${ }^{*}$
}

1Joint Department of Biomedical Engineering, The University of North Carolina and North Carolina State University, Chapel Hill, North Carolina 27599, USA ${ }_{2}$ Eshelman School of Pharmacy, University of North Carolina, Chapel Hill, NC 27599, USA 3Department of Microbiology and Immunology, University of North Carolina-Chapel Hill, Chapel Hill, North Carolina 27599, USA 4Marsico Lung Institute, University of North Carolina at Chapel Hill, Chapel Hill, North Carolina 27599, USA

\section{* These authors contributed equally to this work}

Address correspondence to: papadopoulou@unc.edu (VP), seconlon@email.unc.edu (SER)

Bacterial biofilms, often associated with chronic infections, respond poorly to antibiotic therapy and frequently require surgical intervention. Biofilms harbor persister cells, metabolically indolent cells, which are tolerant to most conventional antibiotics. In addition, the biofilm matrix can act as a physical barrier, impeding diffusion of antibiotics. Novel therapeutic approaches frequently improve biofilm killing, but usually fail to achieve eradication. Failure to eradicate the biofilm leads to chronic and relapsing infection, associated with major financial healthcare costs and significant morbidity and mortality. We address this problem with a two-pronged strategy using 1) antibiotics that target persister cells and 2) ultrasound-stimulated phase-change contrast agents (US-PCCA), which improve antibiotic penetration.

We previously demonstrated that rhamnolipids, produced by Pseudomonas aeruginosa, could induce aminoglycoside uptake in gram-positive organisms, leading to persister cell death. We have also shown that US-PCCA can transiently disrupt biological barriers to improve penetration of therapeutic macromolecules. We hypothesized that combining antibiotics which target persister cells with US-PCCA to improve drug penetration could eradicate methicillin resistant $S$. aureus (MRSA) biofilms. Aminoglycosides alone or in combination with US-PCCA displayed limited efficacy against MRSA biofilms. In contrast, the anti-persister combination of rhamnolipids and aminoglycosides combined with US-PCCA dramatically reduced biofilm viability, frequently culminating in complete eradication of the biofilm. These data demonstrate that biofilm eradication can be achieved using a combined approach of improving drug penetration of therapeutics that target persister cells.

\section{Introduction}

S. aureus is one of the most important human bacterial pathogens and in 2017 was the cause of 20,000 bacteremia deaths in the US alone ${ }_{1}$. Infections range from minor skin and soft tissue infections (SSTI), implanted device infections to more serious infections such as

43 osteomyelitis, endocarditis and pneumonia 2,3. In addition to the high degree of mortality, chronic and relapsing $S$. aureus infections are common and associated with significant morbidity. This is 
due to frequent treatment failure of $S$. aureus infections. This is best illustrated by SSTIs, with some studies suggesting treatment failure rates as high as $45 \%$ and a recurrence rate of $70 \% 4$. Importantly the failure of antibiotic therapy cannot be adequately explained by antibiotic resistance 1 . Failure to clear the infection leads to a need for prolonged antibiotic therapies, increased morbidity and mortality, increased likelihood of antibiotic resistance development as well as an enormous financial healthcare burden.

$S$. aureus forms biofilms, bacterial cells embedded in a self-produced extracellular matrix, which act as a protective barrier from the host immune response and other environmental assaults. Biofilms expand up to $1200 \mu \mathrm{m}$ in thickness when attached to indwelling devices such as catheters 5. Non-surface attached biofilms, in chronic wounds and chronic lung infections, harbor smaller non-surface attached cell aggregates ranging from 2-200 $\mu \mathrm{m}$ in diameter 5,6. These biofilm aggregates are often surrounded by inflammatory immune cells such as neutrophils and embedded in a secondary host produced matrix such as mucus, pus or wound slough 7. Consequently, biofilm-embedded cells have limited access to nutrients and oxygen and are coerced into a metabolically indolent state 8.

It has long been appreciated that biofilms respond poorly to antibiotics 7,9-12. Most conventional bactericidal antibiotics kill by corrupting ATP-dependent cellular processes; aminoglycosides target translation, fluoroquinolones target DNA synthesis, rifampicin targets transcription and $\beta$-lactams and glycopeptides target cell wall synthesis 13,14 . Cells that survive lethal doses of antibiotics in the absence of a classical resistance mechanism are called antibiotic tolerant persister cells 15 . Biofilms are made up of a high proportion of persister cells 15 18. They are distinct from resistant cells as they cannot grow in the presence of the drug. However, once the drug is removed, persisters grow and repopulate a biofilm and cause a relapse in infection 13. Anti-persister antibiotics which kill independently of the metabolic state of the cell are more effective against biofilms than conventional antibiotics 19-22. Tobramycin, an aminoglycoside that requires active proton motive force (PMF) for uptake into the cell is inactive against non-respiring cells, anaerobically growing cells, small colony variants and metabolically inactive cells within a biofilm 20. We previously reported that rhamnolipids, biosurfactants produced by $P$. aeruginosa, permeabilize the $S$. aureus membrane to allow PMF-independent diffusion of tobramycin into the cell 20,22. This combination of tobramycin and rhamnolipids $(\mathrm{TOB} / \mathrm{RL}$ ) rapidly sterilized in vitro planktonic cultures as well as non-respiring cells, anaerobically growing cells and small colony variants. However, despite this potent antipersister activity, TOB/RL reduced biofilm viability by $\sim 3$-logs but failed to achieve eradication 20 . Notwithstanding the promise of this strategy, eradication of biofilms is arduous, even in vitro, indicating that factors other than the metabolic state of the biofilm-embedded cells are impeding 80 therapy.

The biofilm matrix can act as a physical barrier to drug penetration. Penetration of oxacillin, vancomycin, cefotaxime, chloramphenicol and aminoglycoside antibiotics are impeded to some extent into $S$. aureus biofilms 23-25. Consequently, novel methods of drug delivery into

84 biofilms is a growing area of interest. Ultrasound is a safe, commonplace, portable and relatively 85 inexpensive modality typically used in medical imaging. This imaging capability has been 86 expanded through the use of intravenously administered microbubbles as a contrast agent. 87 These microbubbles are also used in a growing number of therapeutic applications to enhance 
biological effects, which include transdermal drug delivery 26 and transient permeabilization of the blood brain barrier 27.

When exposed to an ultrasound wave, gas-filled microbubbles in solution will oscillate, with the positive pressure cycle resulting in compression and the negative pressure cycle causing the bubble to expand. In an ultrasound field, microbubbles experience stable cavitation (continuous expansion and contraction) at lower pressures or inertial cavitation (violent collapse of the bubble) at higher pressures 28 . Stable cavitation results in microstreaming; fluid movement around the bubble which induces shear stress to nearby structures (such as biofilms). At higher pressures, inertial cavitation can result in a shockwave, producing high temperatures at a small focus, and create microjets from the directional collapse of the bubble which can puncture host cells and disrupt physical barriers 29 . Both of these pressure regimes have potential for therapeutic applications of ultrasound-mediated microbubble cavitation. Despite the potential of microbubbles to enhance drug delivery, their size (typically 1-4 micron in diameter) and short half-life once injected into solution may limit penetration and subsequent

102 disruption of biofilms.

103

We hypothesized that phase change contrast agents (PCCA), submicron liquid particles (typically 100-400 nanometers in diameter) may be better equipped to penetrate a biofilm.

106 PCCAs generally consist of a liquid perfluorocarbon droplet stabilized by a phospholipid shell. With appropriate ultrasound stimulation, PCCA can convert from the liquid phase to gas, generating a microbubble in their place (Fig. 1a). This process of "acoustic droplet vaporization" (ADV) may enhance drug penetration into biofilms as microbubbles over-expand before reaching their final diameter. Prior to activation, these particles are significantly more stable than microbubbles, with the potential to diffuse into biofilms due to their small size (Fig. 1b). Additionally, with continued ultrasound application, the resulting microbubbles can generate microstreaming, shear stress and microjets as they undergo cavitation (Fig. 1b). We hypothesized that PCCA, in combination with ultrasound (US-PCCA) and antibiotics that target persister cells is a novel biofilm eradication strategy.

\section{Results}

We first identified drugs with efficacy against biofilms. Antibiotics were chosen based on clinical relevance or previously reported anti-biofilm efficacy in vitro. Mature MRSA biofilms (USA300 LAC) were cultured for $24 \mathrm{~h}$ in tissue culture treated plates before the addition of antibiotics. Following $24 \mathrm{~h}$ of drug treatment, biofilms were washed and survivors were enumerated by plating. Tobramycin, mupirocin, vancomycin, and linezolid all caused a significant reduction in surviving biofilm cells (Fig. 2a). In contrast, levofloxacin and gentamicin showed no efficacy against biofilms at clinically achievable concentrations found in serum $\left(\mathrm{C}_{\max }\right)$ 24,25 (Fig. 2a).

Next, we tested the ability of 30 second (s) US-PCCA treatment to potentiate tobramycin efficacy. Previous studies have indicated that negatively charged components of the biofilm matrix such as extracellular DNA and certain components of polysaccharides impede penetration of positively charged aminoglycosides such as tobramycin 25,30,31. We hypothesized that US-PCCA might improve tobramycin penetration into biofilms and increase its efficacy. Mature biofilms were washed and transferred to a custom-built temperature-controlled $37^{\circ} \mathrm{C}$ water bath alignment setup. Tobramycin and PCCAs were added and ultrasound applied at a 
132 range of rarefactional pressures $(300-1200 \mathrm{kPa})$. We found that tobramycin efficacy was 133 significantly enhanced at pressures of 300,600 and 1200 but not $900 \mathrm{KPa}$ in the presence of 134 PCCAs (Fig. 2b-c). We confirmed that the addition of PCCA in the absence of ultrasound had no impact on biofilm viability. Similarly, we anticipated that ultrasound alone, in the absence of PCCA would be ineffective, however $1200 \mathrm{kPa}$ did cause a small but significant reduction in surviving cells in the absence of PCCA (Fig. 2b), indicating that potentiation seen at the highest pressure $(1200 \mathrm{kPa})$ may not be entirely attributable to PCCA activity. In order to investigate the potentiation effects of PCCA specifically in the regime below ultrasound-alone effects, the higher pressures (900 and $1200 \mathrm{kPa}$ ) were not evaluated further and the duty cycle lowered to $10 \%$ for subsequent experiments. The lower pressures, 300 and $600 \mathrm{kPa}$, in combination with PCCA were determined to be most effective at potentiating tobramycin efficacy. This is consistent with our previous findings where lower pressures (above the ADV threshold) resulted in more persistent cavitation activity during a 30s ultrasound exposure and was consistently greatest at macromolecule drug delivery across colorectal adenocarcinoma monolayers 32.

Next, we tested the ability of US-PCCA to potentiate mupirocin, vancomycin and linezolid/rifampicin. Mupirocin is a carboxylic acid topical antibiotic commonly used to treat $S$. aureus infections that binds to the isoleucyl-tRNA and prevents isoleucine incorporation into proteins 33 . US-PCCA caused a very slight increase in mupirocin killing (41\% increase in killing) that was statistically significant but of questionable biological significance (Fig. 2a-b).

Vancomycin is a glycopeptide that is the frontline antibiotic to treat MRSA infections. This antibiotic acts by binding to the D-Ala-D-ala residues of the membrane bound cell wall precursor, lipid II, preventing its incorporation and stalling active peptidoglycan synthesis 34 . Importantly, some studies have indicated that vancomycin penetration is impeded into biofilms 24. US-PCCA potentiated vancomycin killing of biofilm-associated cells by $93 \%$ (Fig. 3a-b), likely by improving penetration. Notably, potentiation of vancomycin was seen with the $\mathrm{C}_{\max } 35$ indicating that at a clinically relevant concentration, US-PCCA has the capacity to improve biofilm killing of the front-line antibiotic used to treat MRSA infections.

Linezolid is an oxazolidinone protein synthesis inhibitor that is sometimes combined with

160 the transcriptional inhibitor, rifampicin, for the treatment of $S$. aureus infections $36,37$.

161 Linezolid/rifampicin reduced viable cells within the biofilm by almost 3-logs but was not

162 significantly potentiated by US-PCCA. This suggests that US-PCCA has the ability to potentiate

163 some conventional antibiotics but not others. It is possible that US-PCCA does not potentiate

164 the killing of mupirocin and linezolid/rifampicin because the penetration of these drugs is not

165 impeded into biofilms.

Although the increased killing of biofilm-associated cells with conventional antibiotics

168 in the biofilm are surviving and thus impeding biofilm eradication. We predicted that utilizing US-

169 PCCA to increase penetration of drugs active against antibiotic tolerant persister cells could

170 achieve eradication of a biofilm.

171 Daptomycin is a lipopeptide antibiotic which inserts into the cell membrane and disrupts

172 fluid membrane microdomains 38. Daptomycin has potent activity against recalcitrant

173 populations of $S$. aureus, including biofilms 39,40. Daptomycin in combination with linezolid

174 (DAP/LIN) is the treatment recommended for persistent MRSA bacteremia or vancomycin

175 failure in the Infectious Diseases Society of America 2011 MRSA treatment guidelines 41. We 
found that US-PCCA increased DAP/LIN killing of MRSA biofilms by $83 \%$ and $90 \%$ at $300 \mathrm{kPa}$ and $600 \mathrm{kPa}$, respectively (Fig. 4a-b).

Next, we wanted to investigate if US-PCCA could improve efficacy of other drugs with anti-persister activity. Acyldepsipeptides (ADEPs) are activators of the ClpP protease. We previously reported that ADEPs sterilize persisters by activating the ClpP protease and causing the cell to self-digest in an ATP-independent manner 19. ADEP in combination with rifampicin reduced biofilm cells by $>4$-logs in $24 \mathrm{~h}$. US-PCCA significantly potentiated efficacy of ADEP/RIF at $300 \mathrm{kPa}$ but not $600 \mathrm{kPa}$ (Fig. $4 \mathrm{a}-\mathrm{b})$.

Tobramycin combined with rhamnolipids (TOB/RL), has potent anti-persister activity and has eradiated several recalcitrant populations including non-respiring cells, anaerobically growing cells and small colony variants 20 . Despite this potent anti-persister activity, TOB/RL only reduced biofilm viability by $\sim 3$-logs and failed to achieve biofilm eradication 20 . We reasoned that drug penetration might be inhibited into the biofilms and hypothesized that improving penetration could lead to biofilm eradication. Applying US-PCCA in combination with $\mathrm{TOB} / \mathrm{RL}$ increased killing of biofilm cells by $96 \%$ and $85 \%$ at $300 \mathrm{kPa}$ and $600 \mathrm{kPa}$, respectively (Fig. 4a-b). Importantly, half of the biological replicates were eradicated to the limit of detection. Together this data indicates that anti-persister drugs have potent anti-biofilm activity and this can be potentiated further by improving penetration using US-PCCA.

\section{Discussion}

S. aureus biofilms rarely resolve with antibiotic treatment alone and usually require surgical intervention (debridement, drainage, incision) 42. Many antibiotics reduce bacterial burdens within biofilms but eradication represents an arduous challenge even in vitro 5,15 . In this study, we combine two anti-biofilm strategies to achieve eradication (Fig. 5). Biofilm killing by conventional antibiotics with impeded penetration is improved by US-PCCA (Fig.2b-c, Fig.3a-b, Fig. 5), highlighting the therapeutic potential despite falling short of eradication. Targeting biofilms with anti-persister drugs increases efficacy compared to conventional antibiotics but fails to eradicate (Fig. 4a). Combining these approaches greatly improves biofilm killing, and in some cases resulted in eradication of the biofilm (Fig. 4a, Fig. 5).

Antibiotic treatment failure is a complex issue that imposes a heavy burden on global public health. The last new class of antibiotics to be approved by the FDA was in 200343 . Unlike drugs for chronic illnesses that are administered for life (e.g. heart disease, diabetes), antibiotic regimens are comparatively short, rendering the profitability of antibiotic development low 44 . The void in the drug discovery pipeline makes sensitizing recalcitrant bacterial populations to already approved therapeutics a promising approach. The use of ultrasound and cavitationenhancing agents for antibacterial applications, recently termed "sonobactericide", was first published in 2011 45. While the field is still developing, a significant prospect of therapeutic ultrasound as a mechanical approach to enhance drug efficacy is its compatibility with any molecular therapeutic.

Microbubble oscillation has been shown to cause discrete morphologic changes in a $P$. aeruginosa biofilm 46. Disruption of the physical structure of the biofilm may increase penetration depth of molecules which would otherwise be impeded. Disruption of the biofilm may have other indirect effects on drug efficacy. For example, bacterial biofilms are often hypoxic due to the diffusional distance limit of oxygen. Creating holes in the biofilm may allow oxygen penetration 
and stimulate the metabolic state of the residing persister cells, rendering them sensitive to antibiotics. In support of this, ultrasound in combination with microbubbles has previously been reported to alter the metabolic state of bacterial biofilms 46,47 .

We hypothesized that PCCAs may be more efficient than microbubbles at penetrating biofilms due to their relatively small size and increased stability. The use of US-PCCAs has previously shown to increase vancomycin killing of MRSA biofilms 48 . In contrast to the current study, Hu et al. used perfluoropentane as the perfluorocarbon core, which requires higher pressures than octofluoropropane to vaporize. Even in the absence of an antibiotic, US-PCCA caused a significant reduction in biofilm matrix and metabolic activity measured by threedimensional fluorescence imaging and resazurin 47. The difference in quantification method makes comparison with the previous study difficult (we enumerated bacterial survivors), however our results demonstrate a significant improvement in efficacy using shorter treatment times (30 s vs 5 minutes)49. In addition, the low boiling point PCCAs used in the current study present the advantage that the same low-pressure ultrasound settings can be used for both ADV and subsequent microbubble cavitation. Indeed, this can be achieved with clinically available ultrasound hardware at pressures below the FDA set limits for diagnostic imaging. Additionally, PCCA formulation is a variant of FDA-approved ultrasound contrast microbubbles that have been clinically used for over 25 years in Europe, Asia and USA. This approach may improve the efficacy of existing approved drugs without the additional need for the extensive regulatory approval which accompanies a new molecule. Likewise, as it uses ultrasound parameters that are achievable with clinically available equipment, this has the potential for rapid translation to clinical practice without the need for further technological development.

The ultrasound parameters used in our study mostly varied acoustic pressure and have not yet been optimized for in vivo application. While acoustic pressure is a large contributor to PCCA activation and stimulation, other parameters of frequency, duty cycle, treatment time and PCCA concentration could be further evaluated. Ultrasound is also used clinically for debridement of wounds to disperse biofilms 50. Evaluation of PCCA drug potentiation using lower frequencies and higher intensities typical for this application could give further insight into clinical integration strategies. Future experiments will evaluate the potentiation of antibiotics in a S. aureus mouse skin and soft tissue infection (SSTI). For topical applications such as soft tissue infections, we believe maintaining cavitation activity for the duration of the treatment will be crucial for efficacy, as no new cavitation nuclei will be introduced as would be the case in intravenously administered PCCA (replenished by blood flow).

\section{Methods}

\section{Biofilm assays}

Biofilm assays were performed using the USA300 MRSA strain LAC. It is a highly characterized community-acquired MRSA (CA-MRSA) strain isolated in 2002 from an abscess of an inmate in Los Angeles County jail in California 51. LAC was cultured overnight (18h) in brain heart infusion $(\mathrm{BHI})$ media (Oxoid) in biological triplicates. Each culture was diluted 1:150 in fresh media and

$2602-3 \mathrm{ml}$ was added to the wells of 24-well or 12-well tissue culture treated plates (Costar),

261 respectively. Biofilms were covered with Breathe-Easier sealing strips (Sigma) and incubated at

$262 \quad 37^{\circ} \mathrm{C}$ for $24 \mathrm{~h}$. Biofilms were carefully washed twice with PBS and fresh BHI media containing

263 antibiotics was added. Biofilms were covered and incubated at $37^{\circ} \mathrm{C}$ for $24 \mathrm{~h}$. Biofilms were 
carefully washed twice with PBS before dispersal in a sonicating water bath (5min) and vigorous pipetting. Surviving cells were enumerated by serial dilution and plating. Antibiotics were added at concentrations similar to the $\mathrm{C}_{\max }$ in humans; $10 \mu \mathrm{g} / \mathrm{ml}$ levofloxacin52 (Alfa Aesar), 20 $\mathrm{\mu g} / \mathrm{ml}$ gentamicin 53 (Fisher BioReagents), $58 \mu \mathrm{g} / \mathrm{ml}$ tobramycin 54 (Sigma), $50 \mu \mathrm{g} / \mathrm{ml}$ vancomycin hydrochloride 35 (MP Biomedicals), $15 \mu \mathrm{g} / \mathrm{ml}$ linezolid 55 (Cayman Chemical), 10 $\mu \mathrm{g} / \mathrm{ml}$ rifampicin56 (Fisher BioReagents), $100 \mu \mathrm{g} / \mathrm{ml}$ daptomycin 57 (Arcos Organics), with the exception of the topical antibiotic mupirocin (Sigma) (administered at $100 \mu \mathrm{g} / \mathrm{ml}$ ) and acyldepsipeptide antibiotic (ADEP4) which was added at $10 x$ MIC $(10 \mu \mathrm{g} / \mathrm{ml})$ which previously showed efficacy against $S$. aureus biofilms 19. For daptomycin activity, the media was supplemented with $50 \mathrm{mg} / \mathrm{L}$ of $\mathrm{Ca} 2+$ ions. Where indicated tobramycin was supplemented with $30 \mu \mathrm{g} / \mathrm{ml}$ rhamnolipids22 (50/50 mix of mono- and di-rhamnolipids, Sigma). Where indicated biofilms were treated with PCCA and ultrasound.

\section{PCCA Generation}

Phase change contrast agents were generated as previously reported 58 [Sheeran et al. 2012]. Briefly, 1,2-distearoyl-sn-glycero-3-phosphocholine (DSPC) and 1,2-distearoyl-sn-glycero-3phosphoethanolamine-N-methoxy(polyethylene-glycol)-2000 (DSPE-PEG2000) (Avanti Polar Lipids, Alabaster, AL, USA) were dissolved in 5\% glycerol, 15\% propylene glycol (both from Fisher Chemical, Waltham, MA, USA) in PBS (v/v) at a 1:9 ratio, to a total lipid concentration of $1 \mathrm{mg} / \mathrm{ml}$. Lipid solution $(1.5 \mathrm{ml})$ was dispensed into $3 \mathrm{ml}$ crimp-top vials and degassed under vacuum for 30 minutes and then backfilled with octofluoropropane (OFP) gas (Fluoro Med, Round Rock, TX, USA). The vials were activated by mechanical agitation (VialMix, BristolMyers-Squibb, New York, NY, USA) to generate micron scale OFP bubbles with a lipid coat. The vials containing bubbles were cooled in an ethanol bath to $-11 \mathrm{C}$. Pressurized nitrogen (45 PSI) was introduced by piercing the septa with a needle and used to condense the gaseous octofluoropropane into a liquid, creating lipid-shelled perfluorocarbon submicron droplets (PCCA). Particle size and concentration was characterized the Accusizer Nano FX (Entegris, Billerica, MA, USA).

\section{Ultrasound Experiments}

Ultrasound experiments were conducted in 12 or 24 well tissue culture plates using a custom fabricated water bath ultrasound alignment setup to maintain $37 \mathrm{C}$ during the experiment, similar to a design used previously with cell monolayers 32 . Briefly, alignment guides were positioned above the wells to ensure reproducible transducer placement to the center of each well on top of the biofilm and $10 \mathrm{~mm}$ from their bottom. To limit acoustic reflections and standing waves from the bottom of the well plate, ultrasound gel was applied on the outside bottom of each well before the plate was positioned in the water bath, the bottom of which was lined with acoustic absorber material. The water temperature was maintained at $37 \mathrm{C}$ throughout the experiment by placing the water bath setup on a heated plate and monitored by thermocouple. A $1.0 \mathrm{MHz}$ unfocused transducer (IP0102HP, Valpey Fisher Corp) was characterized via needle hydrophone and driven with an amplified 20- or 40-cycle sinusoidal signal defined on an arbitrary function generator (AFG3021C, Tektronix, Inc.; 3100LA Power Amplifier, ENI) at a pulse-repetition frequency of $5000 \mathrm{~Hz}(10 \%$ or $20 \%$ duty cycle). Peak negative pressures of $300,600,900$ and $1200 \mathrm{kPa}$ were used in the experiments. To avoid ultrasound-alone effects on 
the biofilm, we focused on the lower pressures, 300 and $600 \mathrm{kPa}$, determined most effective at potentiating tobramycin efficacy with PCCA and lowered the duty cycle from 20\% in Fig. 1 to $10 \%$ in Fig. 2-3 as this was shown to have a more modest effect in our prior work and resulted in significant drug delivery 32. Where indicated, $10 \mu$ of PCCA was added to each well $((1.17 \pm$ $0.4) \times 10_{11}$ particles $/ \mathrm{mL}, 0.18 \mu \mathrm{m}$ diameter) and mixed gently by pipetting. The transducer was positioned in the well in the media above the biofilm and ultrasound treatment was applied for 30 seconds. Following treatment, each plate was incubated at $37 \mathrm{C}$ for $24 \mathrm{~h}$ before enumerating survivors (described in detail above).

\section{Statistical information}

318 The averages of $n=3$ biologically independent samples are shown. The error bars represent the standard deviation of the mean. Statistical analysis was performed using Prism 8 (GraphPad) software. One-way ANOVA with Sidak's or Dunnett's multiple comparison test (as indicated in the figure legends). Statistical significance was defined as $P<0.05$.

\section{Acknowledgements}

This work was supported in part by NIH grants R01Al137273 and Cystic Fibrosis Research Grant to B.P.C and R33CA206939 to P.A.D. We are grateful to Nikki J. Wagner, Lauren C. Radlinski and Matthew Wolfgang for technical advice, as well as Brian Velasco for assistance with PCCA preparation.

\section{Competing interests}

330 P.A.D declares that he is a co-inventor on a patent describing the formulation of low boilingpoint perfluorocarbon agents and a cofounder of Triangle Biotechnology, a company that has licensed this patent. Additionally, P.G.D, P.A.D., B.P.C., V.P. and S.E.R. are all co-inventors on a provisional patent describing the use of low boiling-point phase change contrast agents for enhancing the delivery of therapeutics agents to biofilms. Additionally, B.P.C and S.E.R are coinventors on a provisional patent describing the use of rhamnolipids for potentiating antibiotic efficacy. A.E.S declares that she has no competing interests.

\section{References}

1 Kourtis, A. P. et al. Vital Signs: Epidemiology and Recent Trends in Methicillin-Resistant and in Methicillin-Susceptible Staphylococcus aureus Bloodstream Infections - United States. MMWR Morb Mortal Wkly Rep 68, 214-219, doi:10.15585/mmwr.mm6809e1 (2019).

2 Boucher, H. W. \& Corey, G. R. Epidemiology of methicillin-resistant Staphylococcus aureus. Clin Infect Dis 46 Suppl 5, S344-349, doi:10.1086/533590 (2008).

3 Magill, S. S. et al. Changes in Prevalence of Health Care-Associated Infections in U.S. Hospitals. N Engl J Med 379, 1732-1744, doi:10.1056/NEJMoa1801550 (2018).

4 Olaniyi, R., Pozzi, C., Grimaldi, L. \& Bagnoli, F. Staphylococcus aureus-Associated Skin and Soft Tissue Infections: Anatomical Localization, Epidemiology, Therapy and Potential Prophylaxis. Curr Top Microbiol Immunol 409, 199-227, doi:10.1007/82_2016_32 (2017).

5 Bjarnsholt, T. et al. The in vivo biofilm. Trends Microbiol 21, 466-474, doi:10.1016/j.tim.2013.06.002 (2013). 
403

404

405

406

407

408

409

410

411

412

413

414

415

416

417

418

419

420

421

422

423

424

425

426

427

428

429

430

431

432

433

434

435

436

437

438

439

440

441

442

443

444

445

446

447

448

449

450

451

452

25 Goltermann, L. \& Tolker-Nielsen, T. Importance of the Exopolysaccharide Matrix in Antimicrobial Tolerance of Pseudomonas aeruginosa Aggregates. Antimicrob Agents Chemother61, doi:10.1128/AAC.02696-16 (2017).

26 Oberli, M. A., Schoellhammer, C. M., Langer, R. \& Blankschtein, D. Ultrasoundenhanced transdermal delivery: recent advances and future challenges. Ther Deliv 5, 843-857, doi:10.4155/tde.14.32 (2014).

27 Konofagou, E. E. et al. Ultrasound-induced blood-brain barrier opening. Curr Pharm Biotechnol 13, 1332-1345, doi:10.2174/138920112800624364 (2012).

28 Church, C. C. \& Carstensen, E. L. "Stable" inertial cavitation. Ultrasound Med Biol 27, 1435-1437, doi:10.1016/s0301-5629(01)00441-0 (2001).

29 Tzu-Yin, W., Wilson, K. E., Machtaler, S. \& Willmann, J. K. Ultrasound and microbubble guided drug delivery: mechanistic understanding and clinical implications. Curr Pharm Biotechnol 14, 743-752, doi:10.2174/1389201014666131226114611 (2013).

30 Chiang, W. C. et al. Extracellular DNA shields against aminoglycosides in Pseudomonas aeruginosa biofilms. Antimicrob Agents Chemother 57, 2352-2361, doi:10.1128/AAC.00001-13 (2013).

31 Tseng, B. S. et al. The extracellular matrix protects Pseudomonas aeruginosa biofilms by limiting the penetration of tobramycin. Environ Microbiol 15, 2865-2878, doi:10.1111/1462-2920.12155 (2013).

32 Fix, S. M. et al. Ultrasound-Stimulated Phase-Change Contrast Agents for Transepithelial Delivery of Macromolecules, Toward Gastrointestinal Drug Delivery. Ultrasound Med Biol 45, 1762-1776, doi:10.1016/j.ultrasmedbio.2019.02.004 (2019).

33 Hughes, J. \& Mellows, G. Interaction of pseudomonic acid A with Escherichia coli B isoleucyl-tRNA synthetase. Biochem J 191, 209-219 (1980).

34 Healy, V. L., Lessard, I. A., Roper, D. I., Knox, J. R. \& Walsh, C. T. Vancomycin resistance in enterococci: reprogramming of the $D$-ala-D-Ala ligases in bacterial peptidoglycan biosynthesis. Chem Biol 7, R109-119, doi:10.1016/s1074-5521(00)001162 (2000).

35 Barcia-Macay, M., Lemaire, S., Mingeot-Leclercq, M. P., Tulkens, P. M. \& Van Bambeke, F. Evaluation of the extracellular and intracellular activities (human THP-1 macrophages) of telavancin versus vancomycin against methicillin-susceptible, methicillin-resistant, vancomycin-intermediate and vancomycin-resistant Staphylococcus aureus. J Antimicrob Chemother 58, 1177-1184, doi:10.1093/jac/dkl424 (2006).

36 Schwalm, J. D., El-Helou, P. \& Lee, C. H. Clinical outcome with oral linezolid and rifampin following recurrent methicillin-resistant Staphylococcus aureus bacteremia despite prolonged vancomycin treatment. Can J Infect Dis 15, 97-100, doi:10.1155/2004/768765 (2004).

37 Hashimoto, S. et al. Effect of coadministration of rifampicin on the pharmacokinetics of linezolid: clinical and animal studies. J Pharm Health Care Sci 4, 27, doi:10.1186/s40780-018-0123-1 (2018).

38 Grein, F. et al. $\mathrm{Ca}(2+)$-Daptomycin targets cell wall biosynthesis by forming a tripartite complex with undecaprenyl-coupled intermediates and membrane lipids. Nat Commun 11, 1455, doi:10.1038/s41467-020-15257-1 (2020).

39 Lechner, S., Lewis, K. \& Bertram, R. Staphylococcus aureus persisters tolerant to bactericidal antibiotics. J Mol Microbiol Biotechnol 22, 235-244, doi:10.1159/000342449 (2012).

40 Raad, I. et al. Comparative activities of daptomycin, linezolid, and tigecycline against catheter-related methicillin-resistant Staphylococcus bacteremic isolates embedded in biofilm. Antimicrob Agents Chemother 51, 1656-1660, doi:10.1128/AAC.00350-06 (2007). 
Liu, C. et al. Clinical practice guidelines by the infectious diseases society of america for the treatment of methicillin-resistant Staphylococcus aureus infections in adults and children: executive summary. Clin Infect Dis 52, 285-292, doi:10.1093/cid/cir034 (2011).

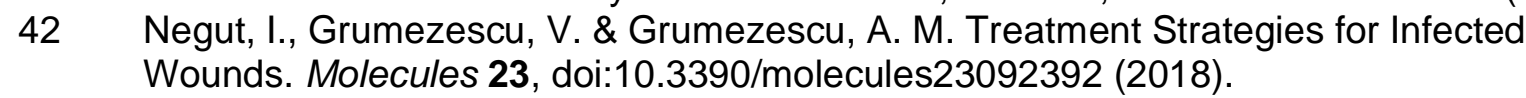

43 Fair, R. J. \& Tor, Y. Antibiotics and bacterial resistance in the 21 st century. Perspect Medicin Chem 6, 25-64, doi:10.4137/PMC.S14459 (2014). Bartlett, J. G., Gilbert, D. N. \& Spellberg, B. Seven ways to preserve the miracle of antibiotics. Clin Infect Dis 56, 1445-1450, doi:10.1093/cid/cit070 (2013).

45 Lattwein, K. R. et al. Sonobactericide: An Emerging Treatment Strategy for Bacterial Infections. Ultrasound Med Biol 46, 193-215, doi:10.1016/j.ultrasmedbio.2019.09.011 (2020).

46 Ronan, E., Edjiu, N., Kroukamp, O., Wolfaardt, G. \& Karshafian, R. USMB-induced synergistic enhancement of aminoglycoside antibiotics in biofilms. Ultrasonics 69, $182-$ 190, doi:10.1016/j.ultras.2016.03.017 (2016).

$47 \mathrm{Hu}$, J. et al. The synergistic bactericidal effect of vancomycin on UTMD treated biofilm involves damage to bacterial cells and enhancement of metabolic activities. Sci Rep 8 , 192, doi:10.1038/s41598-017-18496-3 (2018).

48 Guo, H. et al. Stimulated phase-shift acoustic nanodroplets enhance vancomycin efficacy against methicillin-resistant Staphylococcus aureus biofilms. Int J Nanomedicine 12, 4679-4690, doi:10.2147/IJN.S134525 (2017).

49 . (!!! INVALID CITATION !!! 44).

50 Chang, Y. R., Perry, J. \& Cross, K. Low-Frequency Ultrasound Debridement in Chronic Wound Healing: A Systematic Review of Current Evidence. Plast Surg (Oakv) 25, 21-26, doi:10.1177/2292550317693813 (2017).

51 Kennedy, A. D. et al. Epidemic community-associated methicillin-resistant Staphylococcus aureus: recent clonal expansion and diversification. Proc Natl Acad Sci U S A 105, 1327-1332, doi:10.1073/pnas.0710217105 (2008).

52 Cao, G. et al. Pharmacokinetics and pharmacodynamics of levofloxacin injection in healthy Chinese volunteers and dosing regimen optimization. J Clin Pharm Ther 38, 394-400, doi:10.1111/jcpt.12074 (2013).

53 Lanao, J. M. et al. Pharmacokinetic basis for the use of extended interval dosage regimens of gentamicin in neonates. J Antimicrob Chemother 54, 193-198, doi:10.1093/jac/dkh261 (2004).

54 Burkhardt, O. et al. Once-daily tobramycin in cystic fibrosis: better for clinical outcome than thrice-daily tobramycin but more resistance development? J Antimicrob Chemother 58, 822-829, doi:10.1093/jac/dkl328 (2006).

55 MacGowan, A. P. Pharmacokinetic and pharmacodynamic profile of linezolid in healthy volunteers and patients with Gram-positive infections. J Antimicrob Chemother 51 Suppl 2, ii17-25, doi:10.1093/jac/dkg248 (2003).

56 Peloquin, C. A. et al. Population pharmacokinetic modeling of isoniazid, rifampin, and pyrazinamide. Antimicrob Agents Chemother 41, 2670-2679 (1997).

57 Benvenuto, M., Benziger, D. P., Yankelev, S. \& Vigliani, G. Pharmacokinetics and tolerability of daptomycin at doses up to 12 milligrams per kilogram of body weight once daily in healthy volunteers. Antimicrob Agents Chemother 50, 3245-3249, doi:10.1128/AAC.00247-06 (2006).

501

502

503 
a

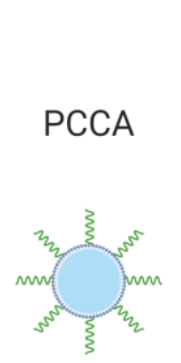

$150 \mathrm{~nm}$

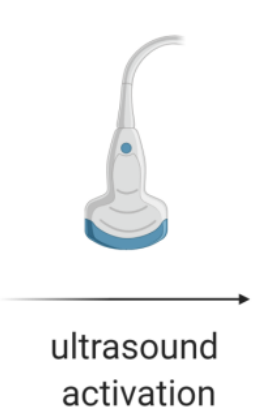

(ADV)

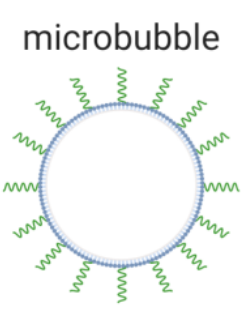

$1 \mu \mathrm{m}$ b

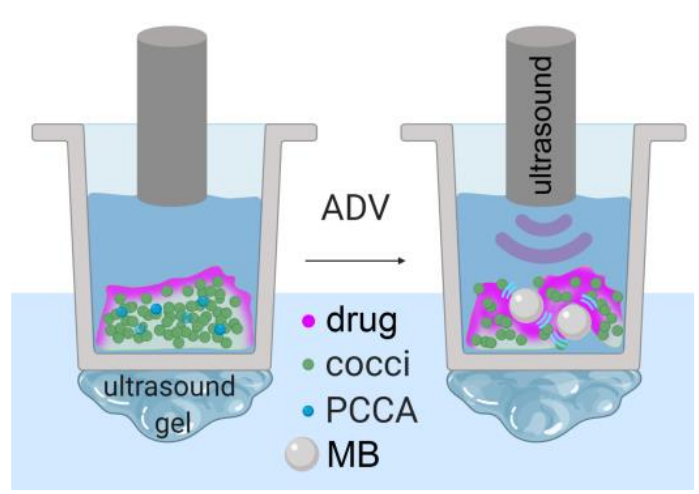

Fig 1. PCCA and ultrasound disrupts biofilm and increases drug penetration. Nanoscale PCCA are in a stable liquid phase. When exposed to ultrasound, the lipid shell containing superheated liquid perfluorocarbon is destabilized, causing the liquid to vaporize (acoustic droplet vaporization, ADV) to the gas phase and expand into a microbubble (a). The stability and small size of PCCA makes them ideal to diffuse into biofilms prior to ultrasound application. Ultrasound stimulation can vaporize PCCA to microbubbles that can physically disrupt biofilms and enhance drug penetration (b). 
a

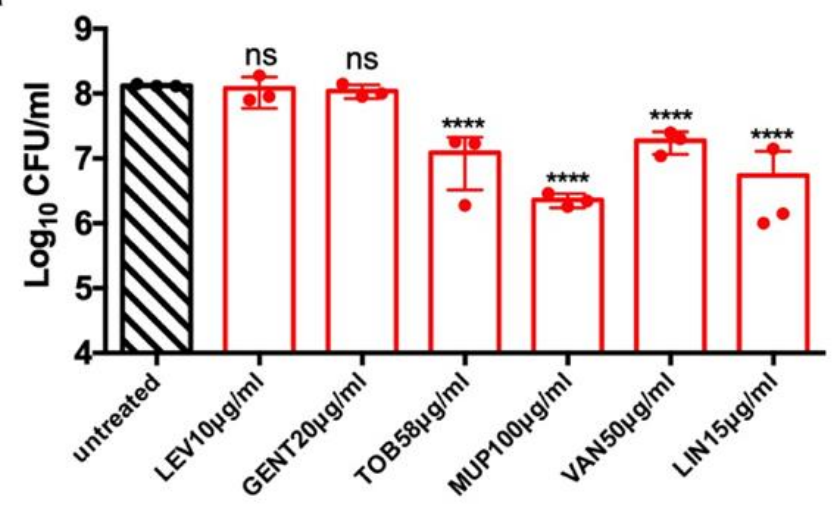

b

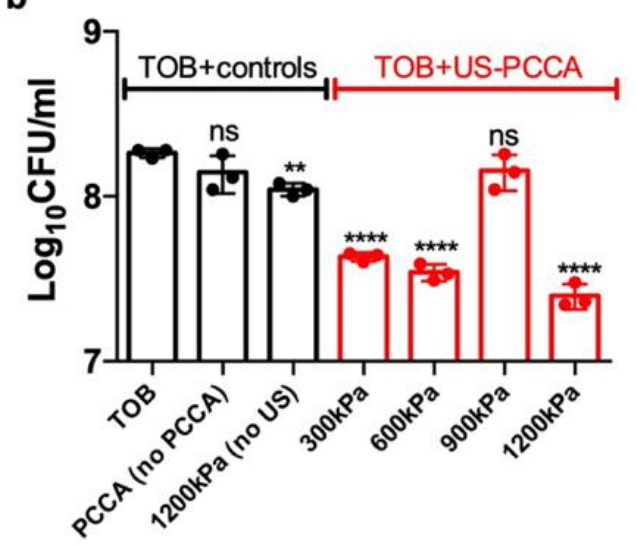

C

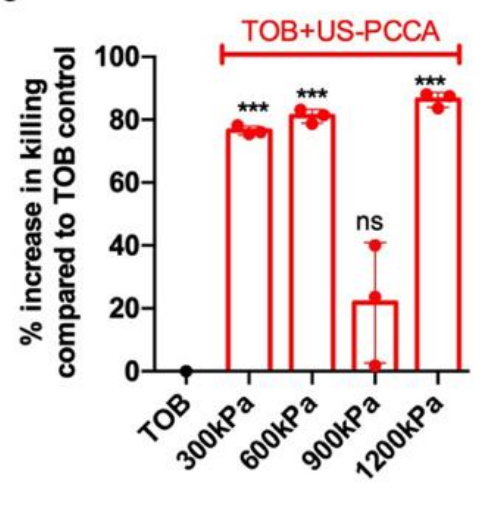

Fig. 2: MRSA biofilms display high tolerance to clinically relevant antibiotics. MRSA strain LAC biofilms were cultured overnight in brain-heart infusion (BHI) media in 12-well tissue culture treated plates. Biofilms were washed and treated with antibiotics (a-c). Where indicated, 12-well plates were transferred to a custom-built temperature-controlled $37^{\circ} \mathrm{C}$ water bath alignment setup. PCCA were added and 30s ultrasound exposure was applied at indicated pressures and $20 \%$ duty cycle (b-c). After $24 \mathrm{~h}$, biofilms were washed, sonicated for disruption and surviving cells were enumerated by serial dilution plating. Survivors were presented as $\log _{10} \mathrm{CFU} / \mathrm{ml}(\mathrm{a}-\mathrm{b})$ or as \% increase in killing compared to tobramycin control (c) (extrapolated from a). The averages of $n=3$ biologically independent samples are shown. The error bars represent the standard deviation. Statistical significance was determined using a one-way analysis of variance (ANOVA) with Dunnett's (a, c) or Sidak's multiple comparison test (b). ${ }^{* *},{ }^{* * *},{ }^{* * * *}$ denotes $P<0.005, P<0.0005, P<0.0001$, respectively. LEV, levofloxacin; GENT, gentamicin; TOB, tobramycin; MUP, mupirocin; VAN, vancomycin; LIN, linezolid, ns, not significant; US-PCCA, ultrasound-stimulated phase change contrast agents. 
a

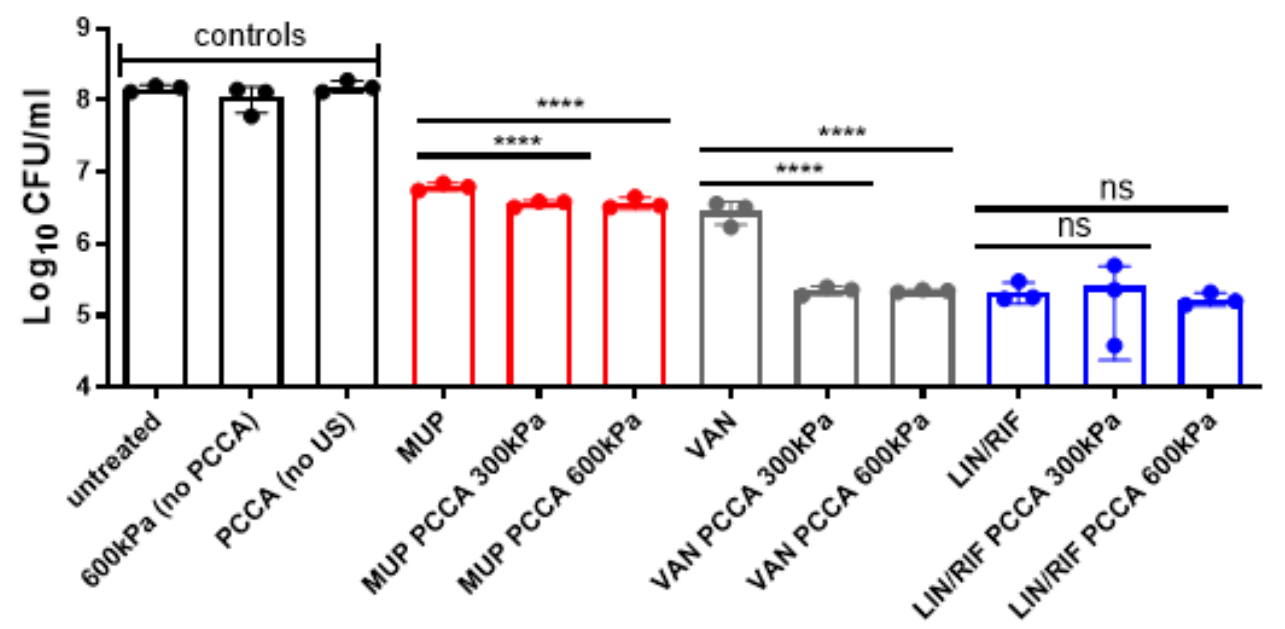

b

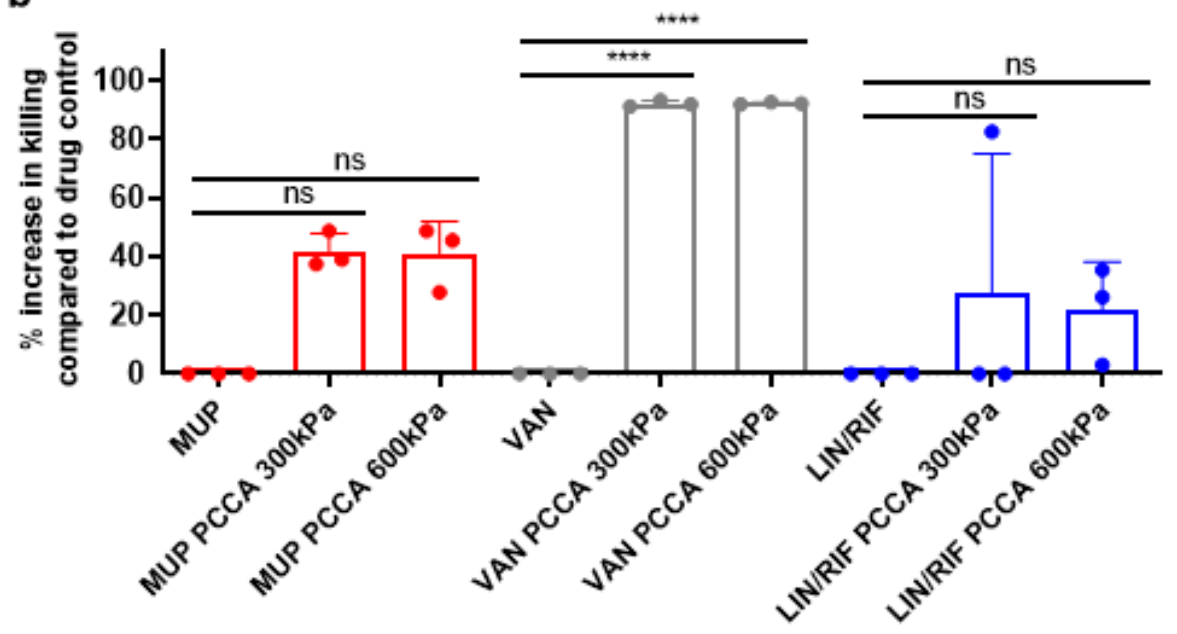

Fig. 3: US-PCCA improve vancomycin killing of MRSA biofilms. MRSA strain LAC biofilms were cultured overnight in brain-heart infusion (BHI) media in 24-well tissue culture treated plates. Biofilms were washed and treated with antibiotics and transferred to a custom-built temperature-controlled $37^{\circ} \mathrm{C}$ water bath alignment setup. PCCA were added and $30 \mathrm{~s}$ ultrasound exposure was applied at indicated pressures and $10 \%$ duty cycle. After $24 \mathrm{~h}$, biofilms were washed, sonicated for disruption and surviving cells were enumerated by serial dilution plating. Survivors were presented as $\log _{10} \mathrm{CFU} / \mathrm{ml}$ (a) or as \% increase in killing compared to antibiotic control (b) (extrapolated from a). The averages of $n=3$ biologically independent samples are shown. The error bars represent the standard deviation. Statistical significance was determined using a one-way analysis of variance (ANOVA) with Sidak's multiple comparison test. **** denotes $P<0.0001$, respectively. MUP, $100 \mu \mathrm{g} / \mathrm{ml}$ mupirocin; VAN, $50 \mu \mathrm{g} / \mathrm{ml}$ vancomycin; LIN, $15 \mu \mathrm{g} / \mathrm{ml}$ linezolid; RIF, $10 \mu \mathrm{g} / \mathrm{ml}$ rifampicin; ns, not significant; US-PCCA, ultrasound-stimulated phase change contrast agents. 
a

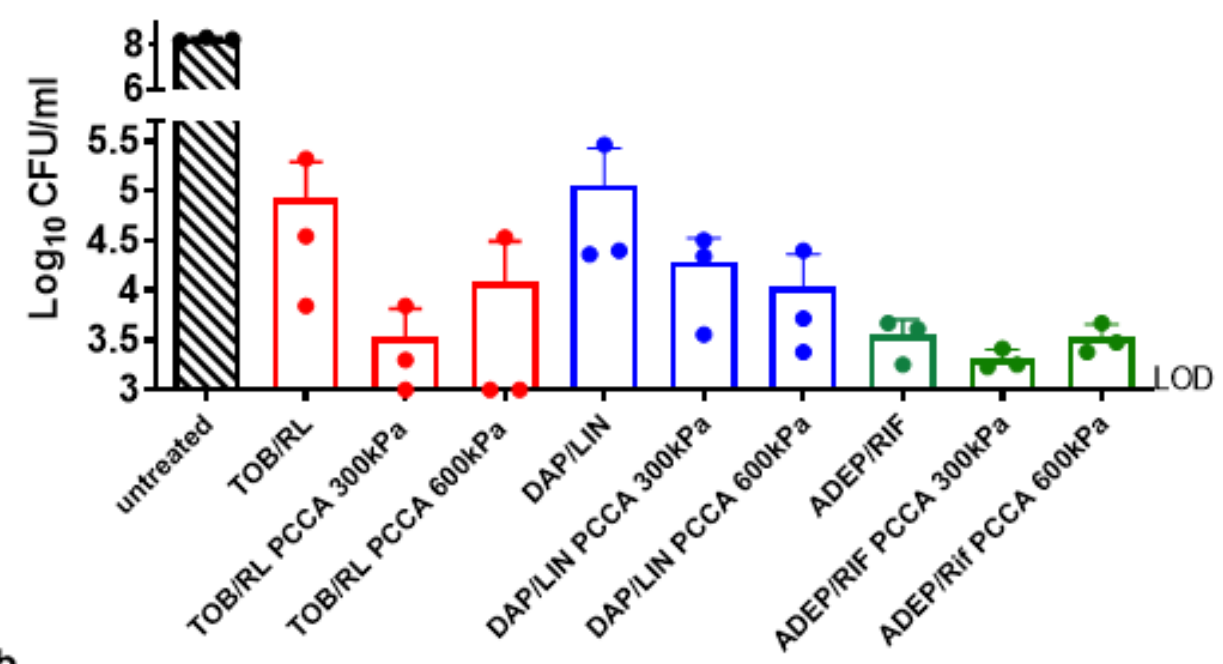

b

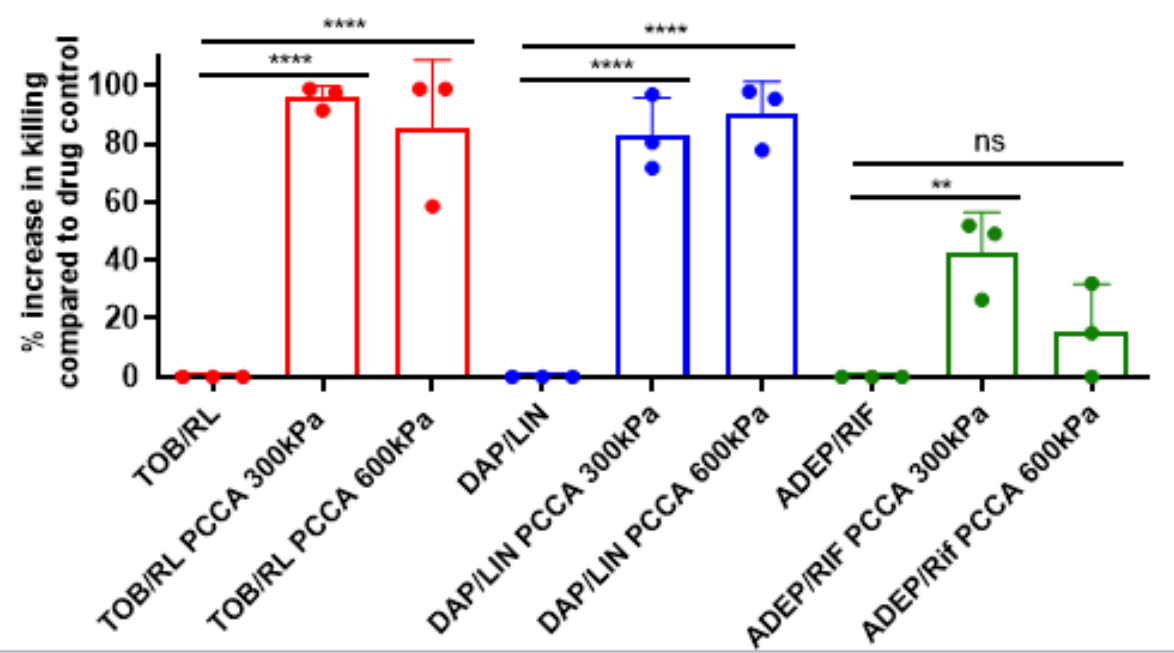

Fig. 4: US-PCCA improves anti-persister antibiotic therapy against MRSA biofilms. MRSA strain LAC biofilms were cultured overnight in brain-heart infusion (BHI) media in 12-well (TOB/RL) or 24-well (all other drugs) tissue culture treated plates. Biofilms were washed and treated with antibiotics and transferred to a custom-built temperature-controlled $37^{\circ} \mathrm{C}$ water bath alignment setup. PCCAs were added and 30 s ultrasound exposure was applied at indicated pressures and $10 \%$ duty cycle. After $24 \mathrm{~h}$, biofilms were washed, sonicated for disruption and surviving cells were enumerated by serial dilution plating. Survivors were presented as $\log _{10} \mathrm{CFU} / \mathrm{ml}$ (a) or as \% increase in killing compared to antibiotic control (b) (extrapolated from a). The averages of $n=3$ biologically independent samples are shown. The error bars represent the standard deviation. Statistical significance was determined using a one-way analysis of variance (ANOVA) with Sidak's multiple comparison test (b). ${ }^{* *},{ }^{* * *}$ denotes $\mathrm{P}<0.005$, $\mathrm{P}<0.0001$, respectively. TOB, $58 \mu \mathrm{g} / \mathrm{ml}$ tobramycin; RL, 30 $\mathrm{gg} / \mathrm{ml}$ rhamnolipids; DAP, $100 \mu \mathrm{g} / \mathrm{ml}$ daptomycin; LIN, $15 \mu \mathrm{g} / \mathrm{ml}$ linezolid; RIF, $10 \mu \mathrm{g} / \mathrm{ml}$ rifampicin; ADEP, $5 \mu \mathrm{g} / \mathrm{ml}$ acyldepsipeptide; ns, not significant; US-PCCA, ultrasound-stimulated phase change contrast agents, LOD; limit of detection. 

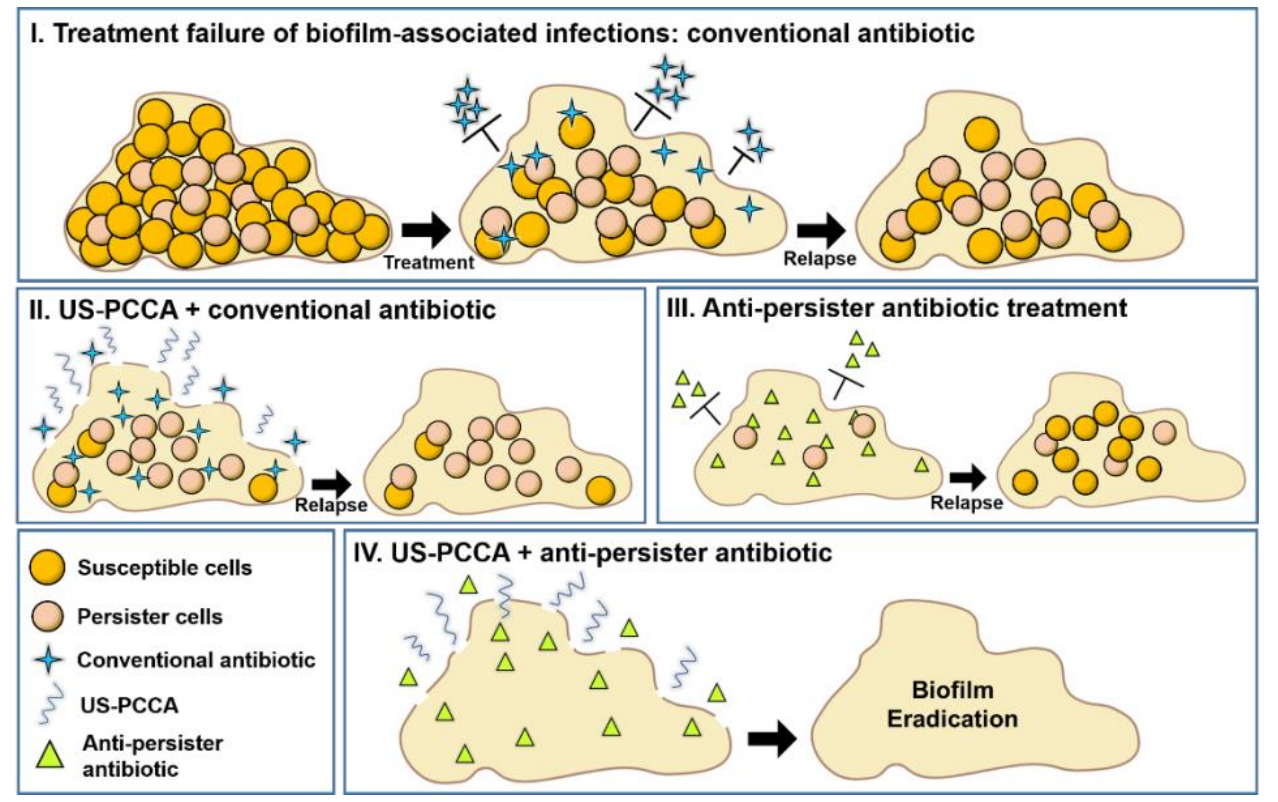

564 Fig. 5: Schematic representing a dual approach to improving antibiotic therapy against $\mathbf{S}$. aureus biofilms. (I) Biofilms display remarkable tolerance to antibiotics. Susceptible cells at the biofilm periphery die (susceptible cells) while less metabolically active cells within the biofilm are tolerant to conventional antibiotics (persister cells). Failure to eradicate the biofilm leads to relapse in infection following removal of the antibiotic. (II) Improving penetration of conventional antibiotics using US-PCCA will improve efficacy of some conventional antibiotics that do not penetrate well through the biofilm matrix. This strategy is futile as it does not improve killing of persister cells and leads to relapse. (III) Targeting biofilms with antibiotics which kill persister cells (anti-persister antibiotics) improves efficacy but if drug penetration is impeded into the biofilm, some persister cells will remain following drug treatment and lead to relapse. (IV) Improving penetration of anti-persister antibiotics into the biofilm can lead to the eradication of all cells within a biofilm and prevent relapse of infection following removal of the antibiotic. 